Influence of confinement on the steady state behavior of single droplets in shear flow for immiscible blends with one viscoelastic component

\author{
R. Cardinaels, K. Verhulst, P. Moldenaers \\ Lab for Applied Rheology and Polymer Processing \\ Department of Chemical Engineering \\ KU Leuven
}

Willem de Croylaan 46, Box 2423, B-3001 Leuven, Belgium

Paula.Moldenaers@cit.kuleuven.be

Publisher's version

Cite as: R. Cardinaels, K. Verhulst, P. Moldenaers, Journal of Rheology, 53(6), pp. 1403-1424 (2009)

The original publication is available at:

http://journalofrheology.org/resource/1/jorhd2/v53/i6/p1403_s1

Copyright: The Society of Rheology 


\title{
Influence of confinement on the steady state behavior of single droplets in shear flow for immiscible blends with one viscoelastic component
}

\author{
R. Cardinaels, K. Verhulst, ${ }^{\text {a) }}$ and P. Moldenaers ${ }^{\text {b) }}$ \\ Department of Chemical Engineering and Leuven Materials Research Center, \\ Katholieke Universiteit Leuven, W. de Croylaan 46, B-3001 Leuven, Belgium
}

(Received 21 April 2009; final revision received 16 August 2009)

\begin{abstract}
Synopsis
By using a counter rotating plate-plate device, single droplets in shear flow have been microscopically studied at confinement ratios ranging from 0.1 to 0.75 . The droplet-to-matrix viscosity ratio was fixed at 0.45 and 1.5 . Results are presented for systems with a viscoelastic Boger fluid matrix or a viscoelastic Boger fluid droplet, at a Deborah number of 1. Although the separate effects of confinement and component viscoelasticity on droplet dynamics in shear flow are widely studied, we present the first systematic experimental results on confined droplet deformation and orientation in systems with viscoelastic components. Above a confinement ratio of 0.3 , wall effects cause an increase in droplet deformation and orientation, similar to fully Newtonian systems. To describe the experimental data, the Shapira-Haber theory [Shapira, M., and S. Haber, Int. J. Multiph. Flow 16, 305-321 (1990)] for confined slightly deformed droplets in Newtonian-Newtonian systems is combined with phenomenological bulk models for systems containing viscoelastic components [Maffettone, P. L., and F. Greco, J. Rheol 48, 83-100 (2004); M. Minale, J. Non-Newtonian Fluid Mech. 123, 151-160 (2004)]. The experimental results are also compared to a recent model for confined droplet dynamics in fully Newtonian systems [M. Minale, Rheol. Acta 47, 667-675 (2008)]. For different values of the viscosity ratio, component viscoelasticity and Ca-number, good agreement was generally obtained between experimental results and predictions of one or more models. However, none of the models can accurately describe all experimental data for the whole range of parameter values. (C) 2009 The Society of Rheology. [DOI: 10.1122/1.3236837]
\end{abstract}

\section{INTRODUCTION}

The properties of immiscible polymer blends are strongly influenced by their twophasic structure. This blend morphology is generated by a combination of deformation, break-up, and coalescence. The final structure depends not only on the component properties and the blend composition but also on the prevailing flow conditions in the processing equipment. Hence, a general understanding of the interrelation between flow and morphology would enable the tailoring of material properties for specific applications.

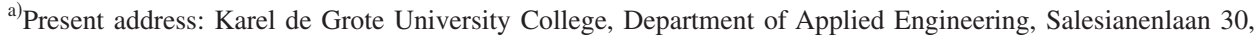
B-2660 Antwerpen, Belgium.

b) Author to whom correspondence should be addressed; electronic mail: paula.moldenaers@cit.kuleuven.be
} 
For dilute blends containing Newtonian components in shear flow, models are available that describe and predict the blend behavior [Tucker and Moldenaers (2002); Guido and Greco (2004)].

However, in order to model polymer processing, additional insight is needed. Primarily, industrially relevant materials are commonly non-Newtonian. Therefore, many experimental studies have been undertaken to elucidate the effects of component viscoelasticity on the droplet dynamics. For steady shear flow, it has clearly been established that for slightly deformed droplets, component viscoelasticity hardly influences the droplet deformation, but it increases the orientation toward the flow direction, especially when the matrix is viscoelastic [Guido et al. (2003a, 2003b); Verhulst et al. (2007a)]. At larger deformations, the experimental data are more scattered. Newtonian droplets in a viscoelastic matrix are observed to be more [Elmendorp and Maalcke (1985); Mighri and Carreau (1998)] or less [Guido et al. (2003b); Sibillo et al. (2005); Verhulst et al. (2007a, 2009a)] deformed than in a Newtonian matrix. Droplet viscoelasticity merely causes a slight reduction in the deformation [Elmendorp and Maalcke (1985); Mighri and Carreau (1998); Lerdwijitjarud et al. (2003, 2004); Sibillo et al. (2005)]. For a blend with a viscosity ratio of 1.5 , Verhulst et al. (2009a) even reported to observe hardly any influence of droplet viscoelasticity for De-numbers up to 17 . The apparent contradictions between the different studies could result from experimental limitations. However, experiments also span a wide range of conditions and, in particular, the viscosity ratio can have a significant effect on the results. In addition, the dimensionless parameters, characterizing the component viscoelasticity, are derived from the small-deformation theory. It was shown that even when they are similar, at least with respect to the undisturbed flow field, the resulting droplet deformations can differ outside the small-deformation limit [Verhulst et al. (2007a)]. Therefore, it may be concluded that, despite the extensive amount of experimental data available, the picture is still not unambiguous.

Small-deformation theories [Greco (2002); Yu et al. (2004)] are able to quantitatively capture the droplet behavior at low flow intensities [Guido et al. (2003a, 2003b); Sibillo et al. (2006a)]. For more deformed droplets, various completely phenomenological [Maffettone and Greco (2004); Minale (2004)] or partially phenomenological [Dressler and Edwards (2004); Yu et al. (2005)] models have been proposed. For steady shear flow, these models have been compared to a certain extent with experimental data [Dressler and Edwards (2004); Maffettone and Greco (2004); Minale (2004); Maffettone et al. (2005); Sibillo et al. (2005); Yu et al. (2005); Verhulst et al. (2007a)]. However, quantitative agreement is mostly limited to moderate droplet deformations. The recent improvements in computational techniques enable sophisticated two-dimensional [Chinyoka et al. (2005); Yue et al. (2005); Chung et al. (2008)] and three-dimensional (3D) [Khismatullin et al. (2006); Aggarwal and Sarkar (2007, 2008)] numerical modeling of droplet deformation in blends with viscoelastic components. These simulations can match the stationary experimental results up to considerably high values of the Ca- and Denumbers [Verhulst et al. (2009a, 2009b)].

A second aspect which is commonly neglected is the fact that in complex processing equipment with many bends and small passages, the effects of the walls on the droplet behavior cannot always be omitted. In addition, microfluidic devices are becoming common practice in emulsion technology. Confinement effects on single droplet dynamics have mainly been studied in complex flows and the goal was often to develop set-ups to control the droplet size distribution. Systematical research in this area is rather limited and restricted to blends with Newtonian components [for a recent review, see Van Puyvelde et al. (2008)]. Experimentally, it was shown that, for single droplets in shear flow, the steady state deformation and the orientation toward the flow direction both 
increase when confining droplets between two walls [Sibillo et al. (2006b); Vananroye et al. (2007)]. Vananroye et al. (2007) showed that the effect of confinement on the droplet behavior significantly depends on the viscosity ratio of the blend. Recently, a phenomenological model for the dynamics of confined Newtonian droplets in a Newtonian matrix has been developed [Minale (2008)]. As long as the droplet shape remains ellipsoidal, good agreement between experimental data and model predictions was obtained [Minale (2008)]. In addition, during the last years, numerical simulations of confined droplet dynamics in shear flow for systems with Newtonian components emerged [Janssen and Anderson (2007); Renardy (2007)]. The effects predicted by these simulations are in agreement with the available experimental results, even for highly confined, sigmoidal droplets and up to the critical conditions for breakup [Janssen and Anderson (2007); Renardy (2007); Vananroye et al. (2008b)]. Therefore, the effect of confinement on the droplet dynamics is well understood for systems containing only Newtonian components. For systems with one viscoelastic component, only preliminary experimental results at a viscosity ratio of 1.5, dealing with the steady [Verhulst et al. (2007b, 2008); Cardinaels et al. (2008a)] and transient droplet behavior [Cardinaels et al. (2007, 2008b)] and droplet breakup [Cardinaels et al. (2008a, 2008b)], are available for confined shear flow.

In this work, the research on the influence of confinement on droplet behavior is extended toward more industrially relevant, i.e., viscoelastic, materials. Droplet deformation and droplet orientation in steady shear flow are microscopically investigated with a counter rotating plate-plate device. The confinement effect is studied for both Newtonian droplets in a viscoelastic matrix and viscoelastic droplets in a Newtonian matrix, both at a viscosity ratio below and above 1 . The experimental droplet deformation results are compared with the predictions of several phenomenological models.

\section{MATERIALS AND METHODS}

\section{A. Materials}

Six different blends are studied in the droplet deformation experiments: the first three blends have a viscosity ratio of 1.5 and the other three have a viscosity ratio of 0.45 . For each viscosity ratio, a blend with a Newtonian droplet in a viscoelastic matrix (blends 2 and 5), one with a viscoelastic droplet in a Newtonian matrix (blends 3 and 6), and a blend that contains only Newtonian components (blends 1 and 4) are used. The latter is used as the reference system. For the Newtonian components, either polyisobutylene (PIB1300) (Parapol from Exxon Chemical) or linear polydimethylsiloxanes (PDMS) (Rhodorsil and Silbione from Rhodia) with different viscosities are used. When PDMS was used as matrix material, it was saturated with low molecular weight PIB (Indopol H50 from BP) in order to avoid droplet shrinkage due to diffusion of PIB into the PDMS matrix [Guido et al. (1999)]. As viscoelastic material, a PIB Boger fluid [BF2, see Verhulst et al. (2007a, 2009a)] was prepared. Rheological characterization of the components was performed with an Advanced Rheometric Expansion System (ARES) rheometer. Within the shear rate range of the droplet deformation experiments, the BF2 Boger fluid has a constant viscosity $\eta$ and constant first normal stress coefficient $\Psi_{1}$. Therefore, the rheological parameters of the viscoelastic fluid are determined by fitting the steady shear data with the second order fluids model. The interfacial tension $\Gamma$ of the different systems was determined by fitting the slow flow droplet deformation to the second order theory of Greco (2002). The experimental temperatures and the corresponding characteristics for both the components and their blends are given in Table I. The properties of the matrix and droplet fluid are denoted with, respectively, $m$ and $d$. 
TABLE I. Component and blend characteristics at the experimental temperatures.

\begin{tabular}{cccccccccc}
\hline \hline Blend & $d / m$ & Droplet & Matrix & $\begin{array}{c}T \\
\left({ }^{\circ} \mathrm{C}\right)\end{array}$ & $\begin{array}{c}\eta_{m} \\
(\mathrm{~Pa} \mathrm{~s})\end{array}$ & $\begin{array}{c}\Psi_{1, d} \\
\left(\mathrm{~Pa} \mathrm{~s}^{2}\right)\end{array}$ & $\begin{array}{c}\Psi_{1, m} \\
\left(\mathrm{~Pa} \mathrm{~s}^{2}\right)\end{array}$ & $\begin{array}{c}\Gamma \\
(\mathrm{mN} / \mathrm{m})\end{array}$ & $\begin{array}{c}\lambda \\
(-)\end{array}$ \\
\hline 1 & N/N & PDMS100-200 & PIB1300 & 25.5 & 83.5 & 0 & 0 & 2.7 & 1.5 \\
2 & N/VE & PDMS30-100 & BF2 & 26.4 & 36.5 & 0 & 197 & 2.0 & 1.5 \\
3 & VE/N & BF2 & PDMS30 & 26.0 & 25.2 & 212 & 0 & 2.2 & 1.5 \\
4 & N/N & PDMS30-60 & PIB1300 & 26.2 & 74.2 & 0 & 0 & 2.5 & 0.45 \\
5 & N/VE & PDMS12,5-30 & BF2 & 26.2 & 37.2 & 0 & 204.5 & 2.0 & 0.45 \\
6 & VE/N & BF2 & PDMS100 & 26.2 & 82.6 & 204.5 & 0 & 1.85 & 0.45 \\
\hline \hline
\end{tabular}

\section{B. Experimental setup}

A counter rotating plate-plate device based on a Paar Physica MCR300, combined with a microscopy setup, is used for the droplet deformation experiments. With this device, the droplet under investigation can be kept in a stagnation plane during flow. This enables simple visualization of the deforming droplet with an optical train consisting of a stereo microscope (Wild M5A) and a high speed digital camera (Basler 1394). Illumination is performed with three energy saving lamps (Philips) to avoid temperature gradients within the sample. Both microscope and camera are mounted on vertically translating stages such that the droplet can be studied in the vorticity-velocity plane (top view) and in the velocity-velocity gradient plane (side view). The combination of both images provides a complete 3D picture of the droplet (see Fig. 1). Quantitative image analysis is performed with automated procedures using the SCION IMAGE software. The temperature in the sample is monitored by means of a thermocouple and could be kept constant within $0.2{ }^{\circ} \mathrm{C}$ by controlling the temperature of the room. Only droplets at a sufficient distance from the edge of the surrounding cup and near the midplane of the gap are used, since deviations from a linear shear flow field [Vrentas et al. (1991)] and asymmetrical wall effects [Shapira and Haber (1990)] could influence the experimental results. The gap spacing is varied between 0.4 and $3 \mathrm{~mm}$. To ensure correct shear rates at the small gaps, the gap and alignment of the plates are checked microscopically before each test. Viscosity experiments at different gaps have been performed to verify the no-slip condition at the walls. More detailed information on the experimental setup and the image analysis protocol is given in Verhulst et al. (2007a).

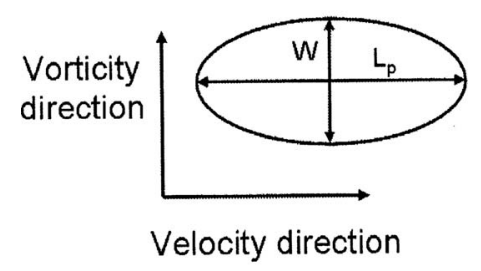

(a) Top view

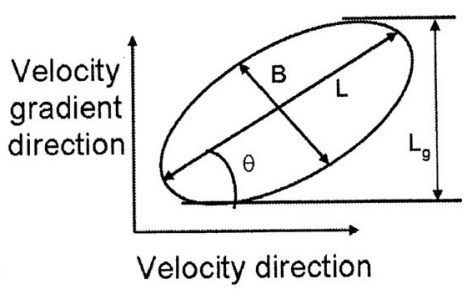

(b) Side view

FIG. 1. Geometrical parameters for a deformed droplet in shear flow. 


\section{ADAPTED SHAPIRA-HABER MODEL FOR VISCOELASTIC COMPONENTS}

When buoyancy and inertia effects can be omitted, the shape and orientation of a single Newtonian droplet in a Newtonian matrix, subjected to bulk flow can be accurately described, up to near-critical conditions, by the phenomenological Maffettone-Minale model [Maffettone and Minale $(1998,1999)]$. In this model, the droplet shape is assumed to be ellipsoidal at all times and it is described by a symmetric, positive definite, and second rank tensor $\mathbf{S}$. The evolution equation for $\mathbf{S}$, resulting from the competing actions of the interfacial tension and the hydrodynamic force, is given by [Maffettone and Minale (1998, 1999)],

$$
\frac{d \mathbf{S}}{d t}-\mathrm{Ca}(\boldsymbol{\Omega} \cdot \mathbf{S}-\mathbf{S} \cdot \mathbf{\Omega})=-f_{1}[\mathbf{S}-g(\mathbf{S}) I]+\mathrm{Ca}_{2}(\mathbf{D} \cdot \mathbf{S}+\mathbf{S} \cdot \mathbf{D}),
$$

with $\mathbf{D}$ as the deformation rate tensor and $\boldsymbol{\Omega}$ as the vorticity tensor. Ca is the well-known capillary number $\left(=\eta_{m} \dot{\gamma} R / \Gamma\right.$, with $\dot{\gamma}$ as the shear rate and $R$ as the droplet radius $)$. At low $\mathrm{Ca}$, the model recovers the linear asymptotic limits of the Taylor theory [Taylor (1934)]. The parameters $\mathrm{g}(\mathbf{S}), f_{1}$, and $f_{2}$ depend on the two governing dimensionless parameters of the problem, namely, $\mathrm{Ca}$ and the viscosity ratio $\lambda\left(=\eta_{d} / \eta_{m}\right)$. The explicit expressions for these parameters can be found in Maffettone and Minale (1998, 1999).

In steady shear flow, effects of the non-Newtonian rheology of the blend constituents only appear at the second order in Ca [Greco (2002)]. Two extensions of the MaffettoneMinale model, which include component viscoelasticity, are available in literature. Both models add a new flow term, which gives its first contribution at second order in $\mathrm{Ca}$, to the right-hand side of Eq. (1). The additional flow term of the Minale model [Minale (2004)] is given in Eq. (2) and that of the Maffettone-Greco model [Maffettone and Greco (2004)] in Eq. (3),

$$
\begin{gathered}
\mathrm{Ca}_{3}\left[(\mathbf{D} \cdot \mathbf{S} \cdot \mathbf{S}+\mathbf{S} \cdot \mathbf{S} \cdot \mathbf{D})-(\mathbf{D} \cdot \mathbf{S}+\mathbf{S} \cdot \mathbf{D}) \frac{1}{3}(\mathbf{S}: \mathbf{I})\right], \\
\mathrm{Ca}(-c) \cdot \mathbf{D} \cdot \operatorname{tr}(\mathbf{S}) .
\end{gathered}
$$

The models recover the second order limit of the Greco model for slow flows [Greco (2002)]. It is assumed that the component rheology obeys the second order fluids model and, therefore, four additional dimensionless parameters appear, as shown by Greco (2002): the Deborah numbers $\operatorname{De}_{d}\left(=\Psi_{1, d} \Gamma / 2 R \eta_{d}^{2}\right)$ and $\operatorname{De}_{m}\left(=\Psi_{1, m} \Gamma / 2 R \eta_{m}^{2}\right)$ and the ratios $\Psi_{m}\left(=-N_{2, m} / N_{1, m}\right.$, with $N_{1}$ and $N_{2}$ as the first and second normal stress differences) and $\Psi_{d}\left(=-N_{2, d} / N_{1, d}\right)$. For the expressions of $f_{1}, f_{2}, f_{3}$, and $c$ as a function of the dimensionless parameters, we refer to the original papers [Maffettone and Greco (2004); Minale (2004)]. Verhulst et al. (2007a) proposed a modification of the expression for the parameter $f_{3}$ in the Minale model that provided better agreement with the experimental data at bulk conditions. This model will be referred to here as the modified Minale model. For the material parameter $\Psi$, a value of 0.1 is assumed here for the Boger fluid, consistent with earlier work [Verhulst et al. (2007a)]. Viscoelastic effects on the droplet deformation are expected to show up for De numbers that are order of magnitude one [Greco (2002)]. In addition, Verhulst et al. (2009a) showed that the effects of viscoelasticity on the steady state deformation in bulk conditions saturate at a De-number of about 2. Therefore, by an appropriate choice of the droplet diameter, the De-number of the viscoelastic phase is kept constant at 1 , a value for which the presence of viscoelastic 
effects is reported [Aggarwal and Sarkar (2007, 2008); Greco (2002); Sibillo et al. (2005); Verhulst et al. (2007a, 2009a)].

The deformation of a droplet in shear flow with inclusion of wall effects has only been studied for systems containing Newtonian components. Recently, the bulk phenomenological Maffettone-Minale model was extended to the case of a generic confined flow [Minale (2008)]. This model, which we will refer to here as the confined Minale model, uses the same evolution equation for $\mathbf{S}$ as the Maffettone-Minale model [Eq. (1)], but altered expressions for $f_{1}$ and $f_{2}$ were derived. Wall effects are taken into account by means of the confinement ratio $2 R / H$, which is the ratio of droplet diameter to gap spacing. For the expressions of $f_{1}$ and $f_{2}$ as a function of $\mathrm{Ca}, \lambda$ and $2 R / H$, we refer to Minale (2008). In the limit of small deformations, this new model leads back to the Shapira-Haber theory [Shapira and Haber (1990)].

For nearly spherical confined droplets, Shapira and Haber (1990) obtained an analytical solution for the droplet shape up to the first order in Ca. If $r, \theta$ and $\varphi$ define a spherical coordinate system located at the droplet origin, the droplet shape is specified by the following equations:

$$
\begin{gathered}
r=R[1+\delta(\theta, \varphi)], \\
\delta(\theta, \varphi)=\mathrm{Ca} \sin \theta \cos \theta \cos \varphi \frac{16+19 \lambda}{8+8 \lambda}\left[1+C_{S} \frac{1+2.5 \lambda}{1+\lambda}\left(\frac{R}{H}\right)^{3}\right] .
\end{gathered}
$$

Here, $r$ is the distance from the center, $\theta$ describes the angular position within the velocity-velocity gradient plane, and $\varphi$ that in the vorticity-velocity gradient plane. $C_{S}$ is a shape coefficient that equals 5.7 for a droplet located at the midplane between the plates [Shapira and Haber (1990)]. Equation (5) for the droplet shape results in the following expression for the droplet deformation parameter $D=(L-B) /(L+B)$ :

$$
D=D_{\text {bulk }}\left[1+C_{S} \frac{1+2.5 \lambda}{1+\lambda}\left(\frac{R}{H}\right)^{3}\right],
$$

with

$$
D_{b u l k}=\mathrm{Ca} \frac{16+19 \lambda}{16+16 \lambda} .
$$

The factor in front of the brackets in Eqs. (5) and (6) describes the bulk deformation following the small-deformation theory of Taylor (1934). Hence, the Shapira-Haber theory predicts that under confinement the droplet deformation in the velocity-velocity gradient plane increases with the factor between brackets, while the $W$-axis remains unaltered, as demonstrated by Eq. (5) with $\varphi=90^{\circ}$.

In the above-mentioned models, either only the effects of confinement or only the effects of viscoelasticity of the components are included. In order to describe the droplet deformation of confined droplets in blends with viscoelastic components, the Taylor bulk deformation part [Taylor (1934)] in Eqs. (5) and (6) is replaced by the deformation part of one of the previously described phenomenological models for bulk droplet deformation in systems with one viscoelastic component [Maffettone and Greco (2004); Minale (2004); Verhulst et al. (2007a)]. This is similar to the approach used by Vananroye et al. (2007), where the Taylor bulk deformation parameter was replaced by that of the Newtonian Maffettone-Minale model [Maffettone and Minale (1998, 1999)]. It should be noted that by using this method, viscoelasticity only influences the final deformation 
TABLE II. Overview of the models used in this work: phenomenological models (1)-(5), analytical model (6), and combination models (7)-(9).

\begin{tabular}{cccc}
\hline \hline & Model & Reference & Component-geometry \\
\hline 1 & Maffettone-Minale & Maffettone and Minale (1998, 1999) & Newtonian-bulk \\
2 & Minale & Minale (2004) & Viscoelastic-bulk \\
3 & Modified Minale & Minale (2004); Verhulst et al. $(2007 \mathrm{a})$ & Viscoelastic-bulk \\
4 & Maffettone-Greco & Maffettone and Greco (2004) & Viscoelastic-bulk \\
5 & Confined Minale & Minale (2008) & Newtonian-confined \\
6 & Shapira-Haber & Shapira and Haber (1990) & Newtonian-confined \\
7 & SH-Maffettone-Minale & Vananroye et al. (2007) & Newtonian-confined \\
8 & SH-Modified Minale & Present work & Viscoelastic-confined \\
9 & SH-Maffettone-Greco & Present work & Viscoelastic-confined \\
\hline \hline
\end{tabular}

through its effect on the bulk deformation, but it is not explicitly taken into account in the correction factor for wall effects. The applicability of this simple approach is discussed in the next sections.

An overview of the terminology and applicability of the different models used in this work is given in Table II. Models 1-5 are fully phenomenological models, which allow the prediction of the full dynamic droplet behavior, whereas models 6-9 only provide predictions of the steady state droplet deformation. Models 2-4 and 5 are extensions of model 1 for, respectively, blends with viscoelastic fluids or in a confined geometry. The former differs in the additional flow term [see Eqs. (2) and (3)] and the assumptions made to solve the overdetermined system that is obtained by fixing the small deformation limit. Model 3 is merely an adaptation of model 2. Model 6 is an analytical model that is only expected to describe the confined data at small deformations. To extend the results of model 6 to higher deformations and viscoelastic components, ad hoc combinations of models 1, 3, and 4 with model 6 are presented in models 7, 8, and 9. Comparisons between experimental data and the predictions of the models for Newtonian components are well documented in literature [Maffettone and Minale (1998, 1999); Vananroye et al. (2007, 2008a); Minale (2008)]. Therefore, these Newtonian model predictions will be used here as a reference case to study the effect of viscoelasticity of the components.

\section{EXPERIMENTAL RESULTS AND DISCUSSION}

\section{A. Droplet shape in bulk shear flow}

First, the effect of droplet and matrix viscoelasticity on the droplet shape in bulk shear flow is studied. The experimental results are compared with the predictions of several phenomenological models that describe droplet dynamics in blends with one viscoelastic component. Based on this comparison, the most appropriate model is selected as the bulk part in the combined models for droplet deformation of blends with one viscoelastic component in confined shear flow.

The dimensionless droplet axes for a Newtonian droplet in a viscoelastic Boger fluid matrix at a viscosity ratio of 0.45 are shown in Fig. 2, together with the results for the Newtonian reference system at this viscosity ratio. At high Ca-numbers, matrix viscoelasticity clearly reduces the droplet deformation, a well-known result, reported by several authors [Guido et al. (2003b); Sibillo et al. (2005); Verhulst et al. (2007a, 2009a)]. Figure 3 provides the dimensionless droplet axes for a viscoelastic droplet in a Newtonian matrix at a viscosity ratio of 0.45 . The experimental dimensionless droplet axes for 


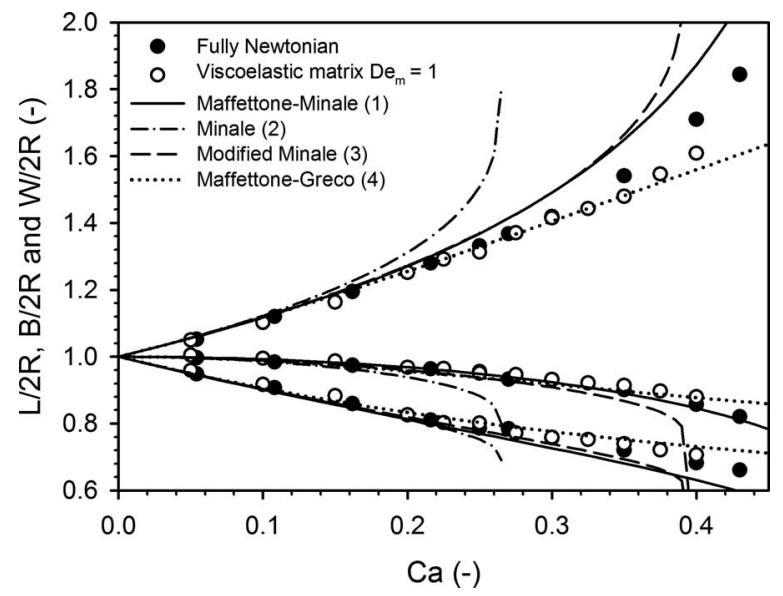

FIG. 2. Dimensionless droplet axes versus Ca-number at bulk conditions $\left(\lambda=0.45\right.$, model predictions at $\mathrm{De}_{m}$ $=1$ ): effect of matrix viscoelasticity.

different De-numbers coincide with those of the Newtonian reference system, showing that, under the conditions of the present study, droplet viscoelasticity has no influence on the droplet deformation. Data on droplet deformation of viscoelastic droplets in a Newtonian matrix are far more scarce than results for the inverse system. At a viscosity ratio of 1.5, Verhulst et al. (2009a) observed no influence of droplet viscoelasticity up to De-numbers as high as 17. This was attributed to the fact that the flow field inside an ellipsoidal droplet is highly rotational [Yue et al. (2005); Verhulst et al. (2009a)]. Therefore, in steady shear flow, effects of droplet viscoelasticity are indeed expected to be minor. However, a slight suppression of the droplet deformation in shear flow, due to the viscoelasticity of the droplet fluid, has been seen by several authors [Elmendorp and Maalcke (1985); Mighri and Carreau (1998); Lerdwijitjarud et al. (2003, 2004); Sibillo et al. (2005)], always at a viscosity ratio of 1 or lower. Droplets that are less viscous than the matrix experience stronger recirculation flows inside the droplet. Moreover, droplet

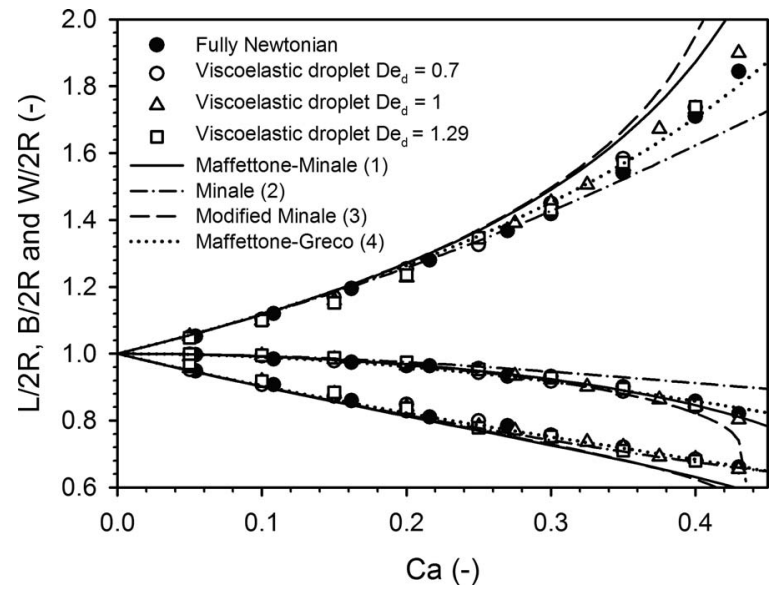

FIG. 3. Dimensionless droplet axes versus Ca-number at bulk conditions $\left(\lambda=0.45\right.$, model predictions at $\mathrm{De}_{d}$ $=1$ ): effect of droplet viscoelasticity. 
deformations are higher than for high viscosity ratio blends, causing the internal flow to deviate more from a pure rotation. Therefore, the viscoelastic stresses are enhanced and the effects of droplet viscoelasticity are expected to be more pronounced at low viscosity ratios. However, since the shear rate inside a droplet differs from the applied shear rate, shear thinning of the used fluids might have influenced some of the reported results. In addition, experimental Newtonian reference data were not always provided.

Up to a Ca-number of 0.3, the Newtonian reference data in Figs. 2 and 3 are well described by the phenomenological Maffettone-Minale model. The predictions of several phenomenological models that describe droplet dynamics in systems with one viscoelastic component are included in the figures. It is clear from Figs. 2 and 3 that, at a viscosity ratio of 0.45 , the predictions of the modified Minale model depend very weakly on either matrix or droplet viscoelasticity. The predicted increase in the droplet deformation, which is also seen in the Minale model predictions for a viscoelastic matrix, is clearly not supported by the experimental data. For the viscoelastic droplets in Fig. 3, both the Maffettone-Greco model and the Minale model predict a reduction in the droplet deformation compared to the Newtonian case, which is clearly in conflict with the present data. Figures 2 and 3 show that at this viscosity ratio, the agreement between experimental data and model predictions is only satisfactory when using the Maffettone-Greco model.

The experimental results at a viscosity ratio of 1.5 are similar to those at a viscosity ratio of 0.45 and can be found in Verhulst et al. (2009a). A comparison between the experimental data and the model predictions was also made at this viscosity ratio. For the sake of brevity, the results will only be mentioned here. The trends predicted by the Minale model are an increased droplet deformation due to matrix viscoelasticity and a pronounced reduction in the droplet deformation due to droplet viscoelasticity, analogous to the results at viscosity ratio 0.45 . However, it can be seen in Verhulst et al. (2009a) that the experimental results behave noticeably different. Similar to the results in Figs. 2 and 3, the Maffettone-Greco model predicts more reduction in droplet deformation than the modified Minale model, independent of the viscoelastic phase. When the matrix is viscoelastic, the best agreement with the experimental data is obtained with the modified Minale model. The data for a viscoelastic droplet with a viscosity ratio of 1.5 are well predicted by the Maffettone-Minale model. Therefore, in the next sections these models are selected as the bulk part in the confined model for systems with a viscoelastic component at a viscosity ratio of 1.5 .

\section{B. Droplet deformation in confined shear flow}

Figure 4 shows the steady state deformation parameter $D$ as a function of the $\mathrm{Ca}$ number for a viscoelastic droplet in a Newtonian matrix together with that of the Newtonian reference blend at a viscosity ratio of 1.5 . The figure contains data for both unconfined and confined droplets, with a confinement ratio $2 R / H$ of 0.11 and 0.74 , respectively. It is clear that the droplet viscoelasticity does not influence the deformation under bulk conditions, in agreement with the results at a viscosity ratio of 0.45 . For both systems during bulk shear flow, the predictions of the Maffettone-Minale model compare well with the data points, up to near-critical conditions. The modified Minale model is also used to describe the bulk data of the blend containing a viscoelastic droplet. The model predicts a significant reduction in the droplet deformation compared to the Newtonian case. It is evident from Fig. 4 and Fig. 4 in Verhulst et al. (2009a) that this reduction is not present in the experimental data.

Introducing confinement significantly increases the droplet deformation, especially at high Ca-numbers (see Fig. 4), similar to what was observed for fully Newtonian systems 


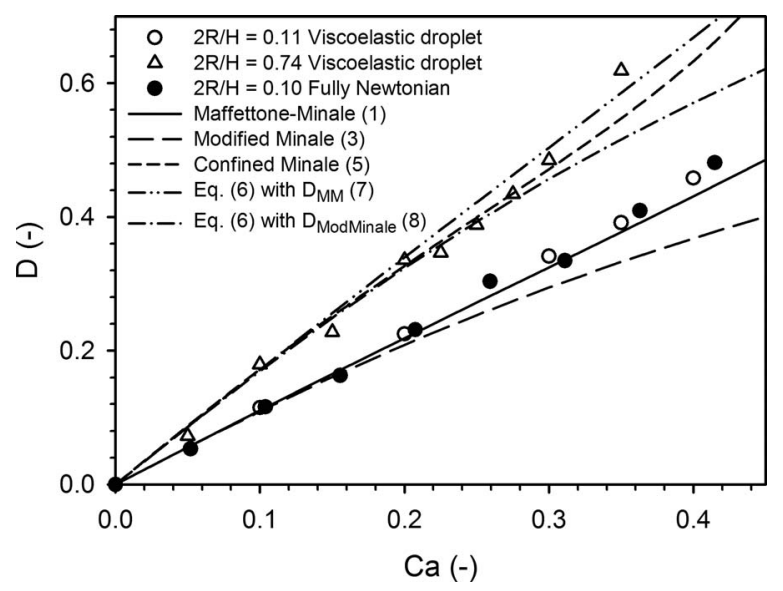

FIG. 4. Deformation parameter for a viscoelastic droplet in a Newtonian matrix at bulk and confined conditions $\left(\lambda=1.5\right.$ and $\left.\mathrm{De}_{d}=1\right)$.

[Vananroye et al. (2007)]. Both the predictions of the confined Minale model and the empirical model consisting of Eq. (6) combined with the Maffettone-Minale bulk deformation parameter are given in Fig. 4 for $2 R / H=0.74$. The latter model predicts slightly higher values for $D$ than the confined Minale model. Good agreement between the data at $2 R / H=0.74$ and the predictions of these fully Newtonian models is obtained. Using the bulk deformation parameter of the modified Minale model in Eq. (6) does, as expected from the poor fit at bulk conditions, not lead to matching results at the highest Canumbers. It is shown in literature that, at a viscosity ratio of 1 , the results of the Newtonian models for confinement agree well with the experimental data for systems that contain only Newtonian components [Vananroye et al. (2007); Minale (2008)]. Therefore, it can be concluded that under the present conditions droplet viscoelasticity does not influence its deformation, even when the droplet is confined between two plates. This conclusion is expected to hold as long as the droplet shape remains ellipsoidal, leading to a mainly rotational flow field inside the droplet.

In Fig. 5 analogous results are provided for a Newtonian droplet in a viscoelastic matrix. It is shown that matrix viscoelasticity substantially reduces the deformation of unconfined droplets. Compared to the results in Fig. 2 for a viscosity ratio of 0.45 , at this higher viscosity ratio of 1.5 , matrix viscoelasticity starts to reduce the droplet deformation at much lower Ca-numbers. The relation between the viscosity ratio and the effects of component viscoelasticity is largely unknown since experimental data at the same De-number or with the same viscoelastic fluid and at different viscosity ratios are scarce in literature. Based on Figs. 2 and 5 and the results in Vananroye et al. (2007) on confinement effects for systems with different viscosity ratios, it is reasonable to state however that, although the viscosity ratio influences the effect of matrix viscoelasticity, its role in the effect of confinement on droplet deformation is more pronounced.

For the viscoelastic matrix case in bulk conditions, there is good agreement between the predictions of the modified Minale model and the experimental data. From a comparison between the model predictions for a system with a viscoelastic matrix at $\mathrm{De}_{m}$ $=1$ and the fully Newtonian system in the range of viscosity ratios from 0.03 to 3 , it became clear that both the modified Minale model and the Maffettone-Greco model predict more reduction in the droplet deformation due to matrix viscoelasticity at the highest viscosity ratios. This trend is in agreement with the experimental results shown in 


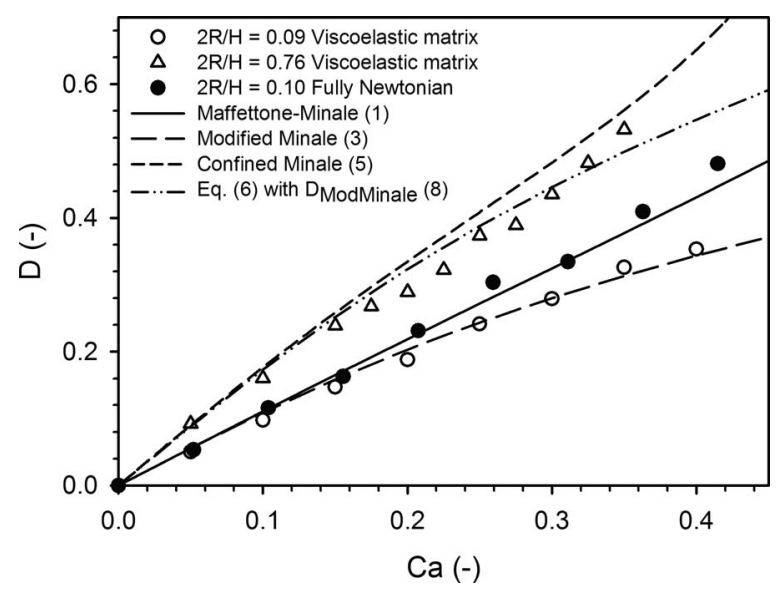

FIG. 5. Deformation parameter for a Newtonian droplet in a viscoelastic matrix at bulk and confined conditions $\left(\lambda=1.5\right.$ and $\left.\mathrm{De}_{m}=1\right)$.

Figs. 2 and 5. In the range of viscosity ratios from 0.03 to 3, the Maffetton-Greco model predicts always lower values for the deformation parameter than the modified Minale model. However, it can be seen from Figs. 2 and 5 that agreement between the experimental results and the different model predictions depends on the viscosity ratio.

Similar to blends with a Newtonian matrix, confinement increases the droplet deformation, as depicted in Fig. 5 for various Ca-numbers. It is shown that the increase in droplet deformation due to wall effects can exceed the inhibiting effect of matrix viscoelasticity at bulk conditions, resulting in a steady droplet deformation that is higher than that observed for a comparable fully Newtonian system in bulk shear flow. However, from a comparison between the experimental data at $2 R / H=0.76$ and the predictions of the confined Minale model, it can be concluded that the deformation is still less than that expected for the Newtonian reference system at a confinement ratio of 0.76 . Thus, also under confinement, matrix viscoelasticity reduces the droplet deformation at this viscosity ratio, at least up to a confinement ratio of 0.75. Agreement with Eq. (6) using the modified Minale bulk deformation parameter for systems with a viscoelastic matrix is satisfactory at this De-number, as shown in Fig. 5.

The deformation parameter as a function of the confinement ratio for several Canumbers is illustrated in Fig. 6 for a blend containing a viscoelastic droplet and in Fig. 7 for a blend with a viscoelastic matrix, both at a viscosity ratio of 1.5 . Wall effects arise above a confinement ratio of approximately 0.3 , irrespective of whether the droplet or the matrix is viscoelastic. The same threshold value was found for completely Newtonian blends [Vananroye et al. (2007)]. For the viscoelastic droplet, as shown in Fig. 6, the experimental data are well described by Eq. (6) with the Maffettone-Minale bulk deformation parameter. However, for this system with a viscosity ratio very close to 1 , the Taylor model [Taylor (1934)] gives nearly the same predictions for the bulk deformation parameter as the Maffettone-Minale model and therefore the original Shapira-Haber theory and the adapted version [Eq. (6) with the bulk Maffettone-Minale deformation parameter] of Vananroye et al. (2007) lead to approximately the same results. Around a viscosity ratio of 1 , differences between the deformation parameter of the Shapira-Haber model and that of the confined Minale model only become apparent at $2 R / H \geq 0.7$ [see Fig. 9 in Minale (2008)]. Hence, the results of the latter model are omitted in order not to overload the graph. It can be concluded that at this viscosity ratio, the predictions of the 


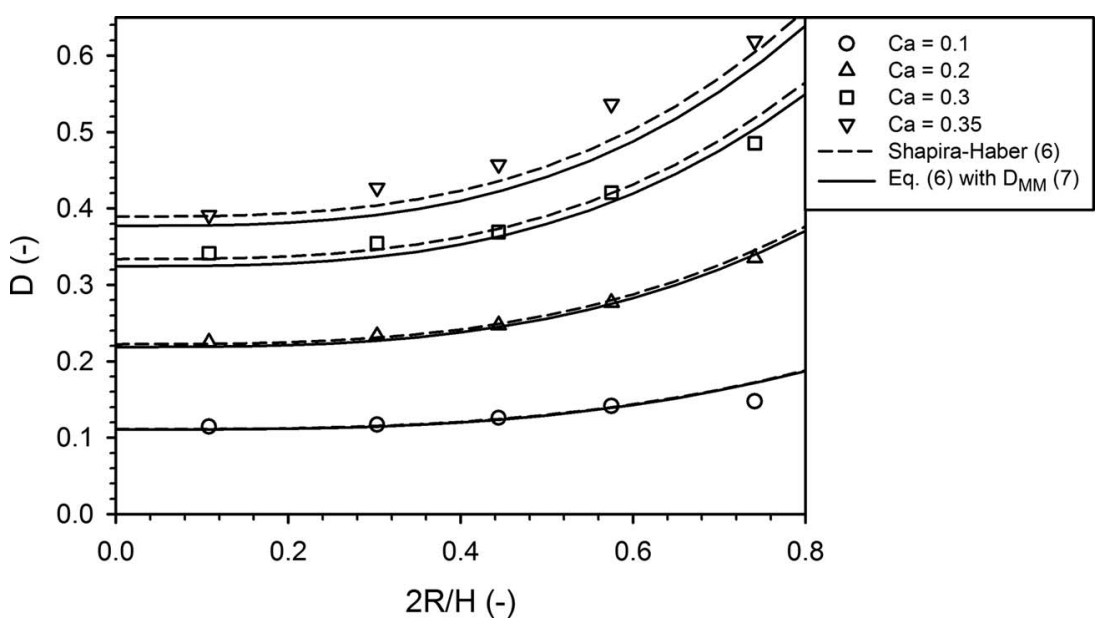

FIG. 6. Deformation parameter versus confinement ratio for a viscoelastic droplet in a Newtonian matrix $(\lambda$ $=1.5$ and $\mathrm{De}_{d}=1$ ).

three available models for the deformation of confined droplets in systems with only Newtonian components give equivalent results. In addition, for the whole range of confinement ratios there is a good agreement between the model predictions and the experimental data for a viscoelastic droplet in a Newtonian matrix, supporting the conclusion that droplet viscoelasticity does not influence the droplet deformation, even for confined droplets.

From Fig. 7, displaying the results for a Newtonian droplet in a viscoelastic matrix, it can be seen that the Newtonian Shapira-Haber theory overestimates the droplet deformation at all confinement ratios, especially for the highest Ca-numbers. Thus, Fig. 7 confirms that viscoelasticity of the matrix reduces the droplet deformation of both confined and unconfined droplets. It is also clear from Fig. 7 that Eq. (6) with the modified Minale bulk deformation parameter for systems with a viscoelastic component adequately predicts the droplet deformation. Therefore, it can be concluded that it is sufficient to

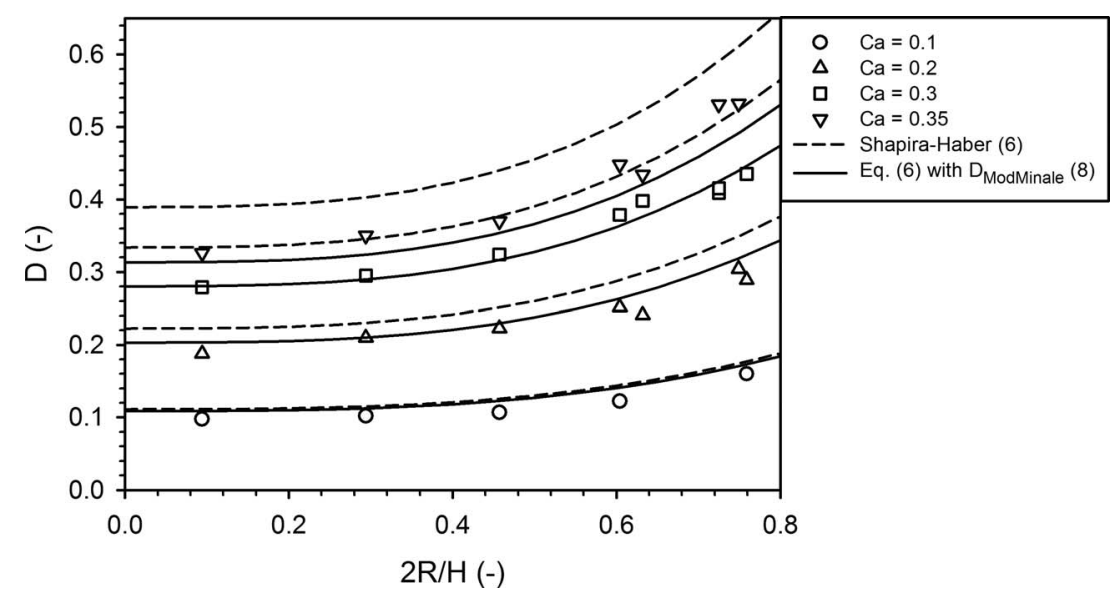

FIG. 7. Deformation parameter versus confinement ratio for a Newtonian droplet in a viscoelastic matrix $(\lambda$ $=1.5$ and $\mathrm{De}_{m}=1$ ). 


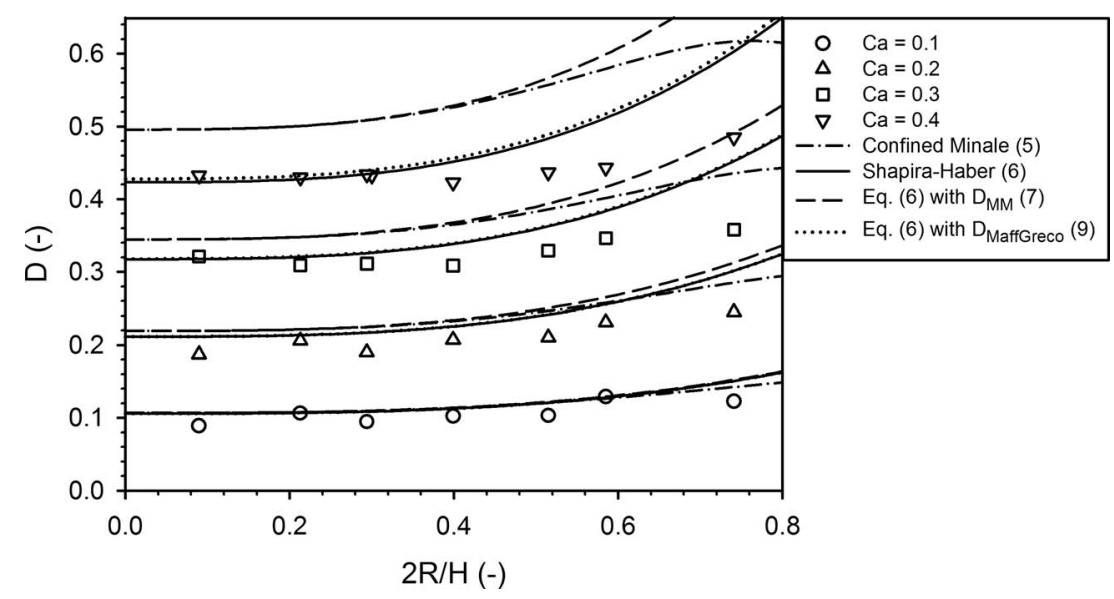

FIG. 8. Deformation parameter versus confinement ratio for a viscoelastic droplet in a Newtonian matrix $(\lambda$ $=0.45$ and $\left.\mathrm{De}_{d}=1\right)$.

include the effect of matrix viscoelasticity in the bulk deformation parameter. The Newtonian Shapira-Haber correction factor for wall effects, which does not include effects of component viscoelasticity, can describe the wall effects on the deformation parameter, at least when the confinement ratio and the component viscoelasticity are not too high.

The deformation parameter as a function of the confinement ratio for systems with a viscosity ratio of 0.45 is shown in Figs. 8 and 9. From Fig. 8, showing the results for a viscoelastic droplet in a Newtonian matrix, it is clear that the confinement effect is much less pronounced at this rather low viscosity ratio. This is in agreement with the results for fully Newtonian systems at a viscosity ratio of 0.31 [Vananroye et al. (2007)]. Model predictions of the confined Minale model for the droplet deformation at a confinement ratio of 0.75 showed that the results for blends of Newtonian fluids with a viscosity ratio of 0.31 and 0.45 are nearly indistinguishable (results not shown). Therefore, it can be concluded that also at this viscosity ratio, the behavior of confined viscoelastic droplets is similar to that of confined Newtonian droplets. The models that recover the Maffettone-

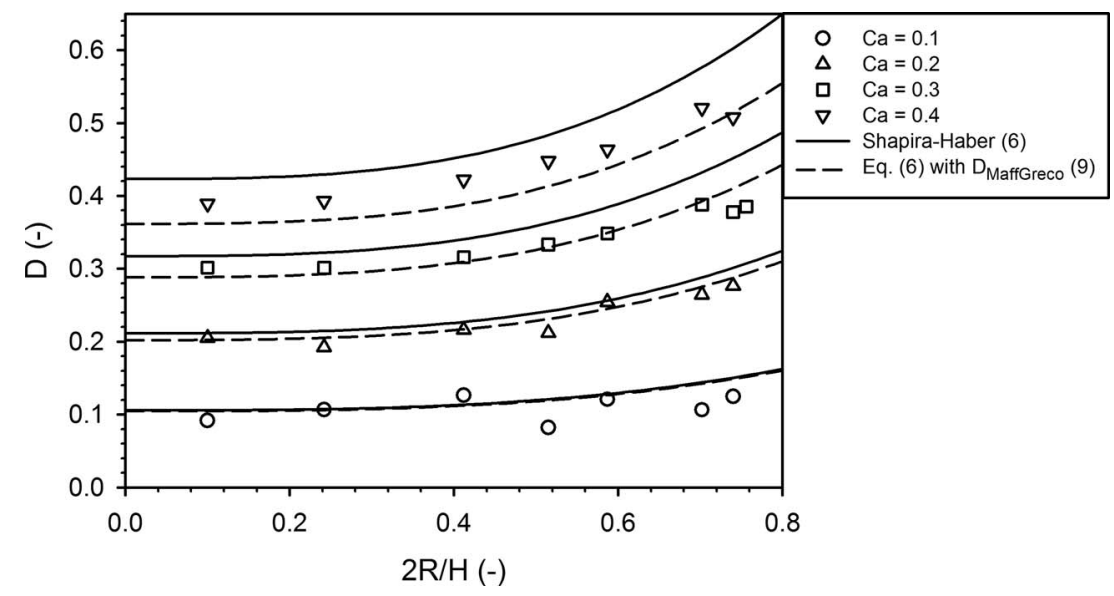

FIG. 9. Deformation parameter versus confinement ratio for a Newtonian droplet in a viscoelastic matrix $(\lambda$ $=0.45$ and $\left.\mathrm{De}_{m}=1\right)$. 


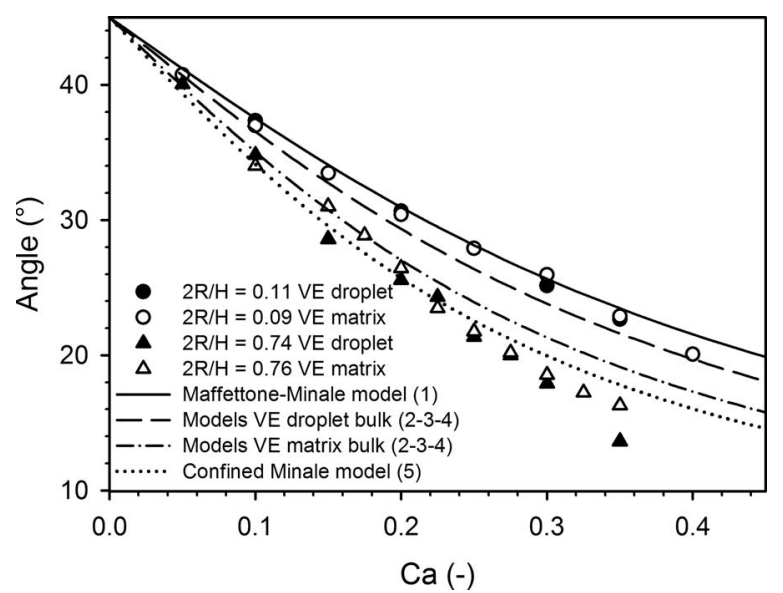

FIG. 10. Orientation angle versus Ca-number at bulk and confined conditions $(\lambda=1.5$ and $\mathrm{De}=1)$.

Minale deformation parameter under bulk conditions overpredict the data at the highest Ca-numbers, as is the case for the bulk data shown in Fig. 3. Although it was shown that the Taylor small-deformation theory is not capable of describing the dimensionless droplet axes outside the small-deformation region [Guido and Greco (2001)], the results for the deformation parameter obtained with this simple first-order model (Shapira-Haber model at $2 R / H=0$ in Fig. 8) nicely coincide with the bulk data. It was indicated by Guido and Greco (2004) that the second-order effects of $\mathrm{Ca}$ on the droplet axes $L$ and $B$ cancel out in the expression for the deformation parameter. Therefore, with the first-order Taylor theory [Taylor (1934)], better results are indeed expected for the deformation parameter than for the droplet axes. Nevertheless, at the viscosity ratio of 0.45 , the Shapira-Haber factor for confinement substantially overpredicts the effect of confinement at the highest Ca-numbers, as can be seen in Fig. 8. This was also the case for fully Newtonian systems at a viscosity ratio of 0.31 [Vananroye et al. (2007)]. In the confined Minale model, the confinement effect is slightly suppressed at the lower viscosity ratios, leading to somewhat better agreement with the data, however, only up to moderate values of the Canumber.

The results for a Newtonian droplet in a viscoelastic matrix at a viscosity ratio of 0.45 are shown in Fig. 9. Under bulk conditions, the deviation between the Newtonian Taylor prediction and the experimental data is clearly less than for the system with a viscosity ratio of 1.5 , indicating that, as already mentioned before, matrix viscoelasticity has less effect at a viscosity ratio of 0.45 (see Fig. 9) than at a viscosity ratio of 1.5 (see Fig. 7). However, at the viscosity ratio of 0.45 , the increase in the droplet deformation with the confinement ratio is more pronounced for this system (see Fig. 9) than for the system with a viscoelastic droplet (see Fig. 8). Therefore, the experimental data for the blend with a viscoelastic matrix fairly follow the trend predicted by the Shapira-Haber factor for wall effects.

\section{Droplet orientation}

The orientation angle, measured with respect to the flow direction (see Fig. 1), for the systems with a viscosity ratio of 1.5 at bulk and confined conditions, is depicted in Fig. 10. At bulk conditions, no effect of the component viscoelasticity on the orientation angle was detected, in agreement with the results in Verhulst et al. (2009a). The orientation 


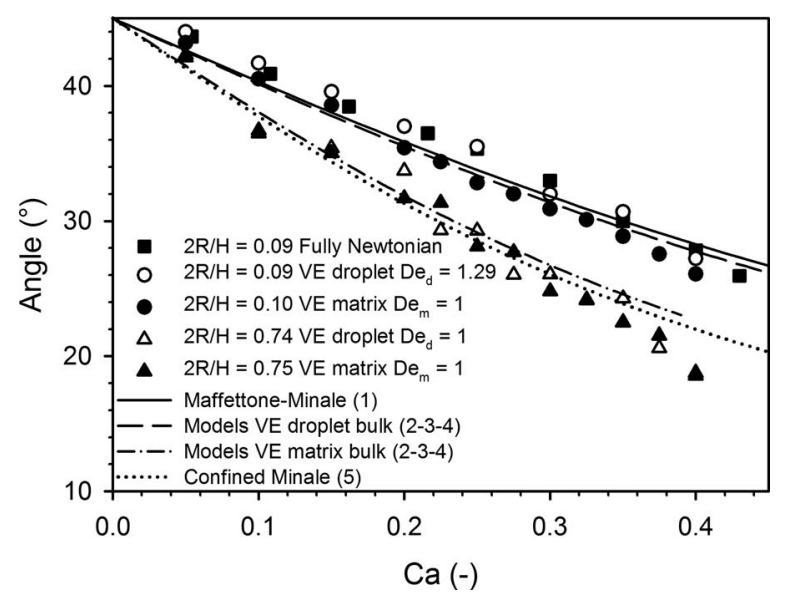

FIG. 11. Orientation angle versus Ca-number at bulk and confined conditions $(\lambda=0.45)$.

angles predicted by the three bulk models for systems with a viscoelastic component (Minale, modified Minale, and Maffettone-Greco models) are exactly the same. The models predict an increased orientation toward the flow direction, especially for a viscoelastic matrix, which is however not observed for the present system. Confining the droplet between two walls causes a more pronounced orientation toward the flow direction, similar to fully Newtonian systems [Vananroye et al. (2007)]. It is demonstrated in Fig. 10 that increasing the Ca-number enhances the orientation effect caused by the presence of the walls. Even for confined droplets, the component viscoelasticity clearly does not influence the droplet orientation. The Shapira-Haber theory predicts no influence of confinement on the orientation angle, as it remains always at $45^{\circ}$ [see Eq. (5)]. The orientation angles obtained with the confined Minale model are in rather good agreement with the experimental data, up to a Ca-number of about 0.25 . Similar results were obtained for fully Newtonian systems [Minale (2008)].

The experimental results and model predictions for the orientation angles at a viscosity ratio of 0.45 are presented in Fig. 11. Also at this viscosity ratio, droplet viscoelasticity does not influence the orientation angle (only the results at $\mathrm{De}_{d}=1.29$ are shown for the sake of brevity). To our knowledge, results for the orientation angle of a viscoelastic droplet are only available in one other study [Sibillo et al. (2005)] at a viscosity ratio of 1. There, an increase in the orientation of the droplets due to droplet viscoelasticity was reported. From Fig. 11, it follows that the orientation angles for a droplet in a viscoelastic matrix are slightly lower than those of a droplet in a Newtonian matrix at a viscosity ratio of 0.45 . A more pronounced influence of matrix viscoelasticity on the droplet orientation was noticed by several authors [Guido et al. (2003a, 2003b); Maffettone and Greco (2004); Sibillo et al. (2005); Verhulst et al. (2007a)], especially at low viscosity ratios, high De- and low Ca-numbers. At a viscosity ratio of 0.45 , the bulk phenomenological models predict an insignificant effect of droplet viscoelasticity on the orientation angle, which agrees well with the experimental data. The effect of matrix viscoelasticity is, however, largely overpredicted. Again, the droplet becomes more oriented toward the flow direction as a consequence of confinement, an effect which is more pronounced at the highest Ca-numbers. Although the confinement effects on the droplet deformation are more moderate at this viscosity ratio than at the higher viscosity ratio of 1.5 (see previous section), the influence of the walls on the droplet orientation is clearly less sensitive to the 


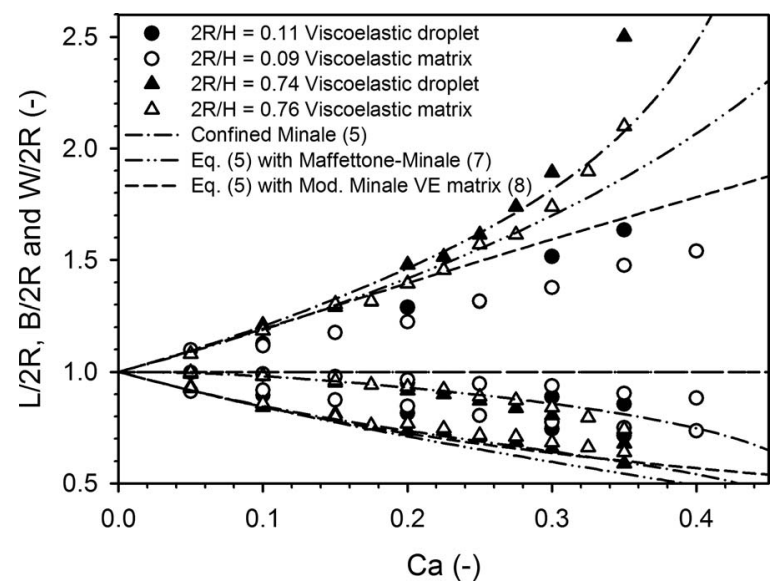

FIG. 12. Droplet axes versus Ca-number at bulk and confined conditions $(\lambda=1.5$ and $\mathrm{De}=1)$.

viscosity ratio. In addition, no systematic difference between the results for the viscoelastic matrix and the viscoelastic droplet is observed. Also for this viscosity ratio of 0.45 , the confined Minale model performs well in predicting the orientation angles of both systems. Therefore, viscoelasticity effects on the orientation angle can be considered to be minor in the present study.

\section{Droplet shape in confined shear flow}

For systems with a viscosity ratio of 1.5 , the results for the three droplet axes at bulk and confined conditions are summarized in Fig. 12. The presence of walls causes a significant extension of the longest droplet axis $L$ and a small reduction in the length of its two other axes $B$ and $W$, similar to fully Newtonian systems [Vananroye et al. (2007)]. This leads to longer and more slender droplets. For both confinement ratios, the droplet deformation in the system with a viscoelastic droplet is higher than in the system with a viscoelastic matrix, a result that is already shown in Figs. 4 and 5 for the deformation parameter.

From Fig. 6 it can be concluded that for systems with a Newtonian matrix, the Shapira-Haber theory is perfectly capable of predicting the deformation parameter $D$ under confined conditions up to rather high Ca-numbers. However, it was shown by Minale (2008) that this theory does not describe the droplet shape correctly, even for ellipsoidal droplets. The expressions for the $L$ and $B$ axes in this first-order theory are symmetric and linear in Ca [Eq. (5)], predicting values for both the $L$ and $B$ axes that are too low at this viscosity ratio. In addition, wall effects on the $W$-axis are not anticipated from the theory, while it is clear from the data that this axis is sensitive to confinement, similar to the $B$ axis. The good agreement for the deformation parameter is caused by the fact that for systems with Newtonian components, the second-order effects in $\mathrm{Ca}$ only occur in the expressions for the droplet axes and not in the deformation parameter [Guido and Greco (2004)]. The predictions for $L$ and $B$ from the Shapira-Haber theory can be improved by replacing the factor outside the brackets in Eq. (5) with that of a bulk model suited for larger deformations, similar to the procedure used for the deformation parameter. Thereto, the bulk models that give the best prediction of the droplet shapes in bulk conditions are used, being the Maffettone-Minale and modified Minale models for, respectively, the viscoelastic droplet and the viscoelastic matrix. As demonstrated in Fig. 

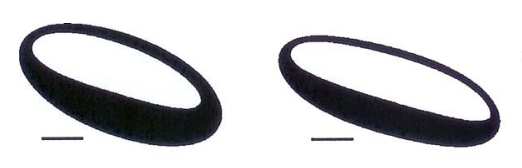

(b) $2 \mathrm{R} / \mathrm{H}=0.58$

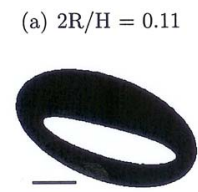

(d) $2 \mathrm{R} / \mathrm{H}=0.09$

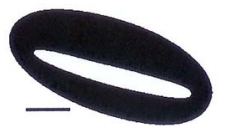

(e) $2 \mathrm{R} / \mathrm{H}=0.60$ (c) $2 \mathrm{R} / \mathrm{H}=0.74$
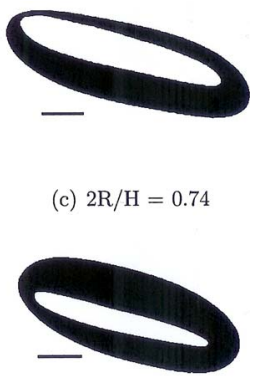

(f) $2 \mathrm{R} / \mathrm{H}=0.76$

FIG. 13. Microscopy images in the velocity-velocity gradient plane for a viscoelastic droplet ( $R=159 \mu \mathrm{m}$, top) and a viscoelastic matrix $(R=148 \mu \mathrm{m}$, bottom) at $\mathrm{Ca}=0.3, \lambda=1.5$, and $\mathrm{De}=1$, indicated scale is $100 \mu \mathrm{m}$.

12, the results for the droplet axes obtained with this adapted Shapira-Haber model are still not as good as the results for the deformation parameter (Figs. 4-7). It is therefore clear that, although the empirical adaptation of the Shapira-Haber theory, by combining it with suitable bulk models, leads to good predictions of the deformation parameter, it cannot adequately capture the complete droplet shape. The confined Minale model, on the other hand, does a rather good job in predicting the three droplet axes of the confined viscoelastic droplet at $2 R / H=0.74$.

The droplet shape in the velocity-velocity gradient plane for increasing confinement ratios is illustrated in Fig. 13 at $\mathrm{Ca}=0.3$. By comparing the pictures in the top (viscoelastic droplet) and bottom (viscoelastic matrix) rows, it can be seen that, for each confinement ratio, the droplet in the system with a viscoelastic matrix is less deformed than that in the system with a viscoelastic droplet. It is also clear from Fig. 13 that above a confinement ratio of approximately 0.7 , the droplet shape starts to become sigmoidal. Since all phenomenological models are derived for ellipsoidal droplet shapes, quantitative agreement between the experimental data and the predictions of the phenomenological models is expected to break down for highly confined droplets. At the same viscosity ratio, this sigmoidal droplet shape is more predominant for viscoelastic droplets. Droplets in a viscoelastic matrix are, however, less deformed and are thus expected to show less sigmoidal droplet shapes.

The droplet axes at a viscosity ratio of 0.45 are presented as a function of the Canumber in Fig. 14. Similar to the results for the deformation parameter, it is clear that the effect of confinement is more pronounced at this lower viscosity ratio when the matrix is viscoelastic. Whereas under bulk conditions droplets are unmistakably less deformed in a viscoelastic matrix than in a Newtonian matrix, the difference between the two systems becomes insignificant at a confinement ratio of 0.75 . The predictions of the confined Minale model are only accurate up to a Ca-number of about 0.28 . The combination of the Shapira-Haber theory with bulk phenomenological models for systems with one viscoelastic component leads to a better agreement with the experimental data. It can therefore be concluded that none of the proposed models can accurately describe all the experimental data at different values of the viscosity ratio, component viscoelasticity, and Ca-number. Therefore, numerical simulations are required to describe the complete droplet dynamics in a wide range of flow and system parameters.

Microscopy images of the droplet shape in the velocity-velocity gradient plane for the systems with a viscosity ratio of 0.45 are shown in Fig. 15. Although for this system the difference in deformation parameter is less drastic, at a confinement ratio $2 R / H=0.75$ a 


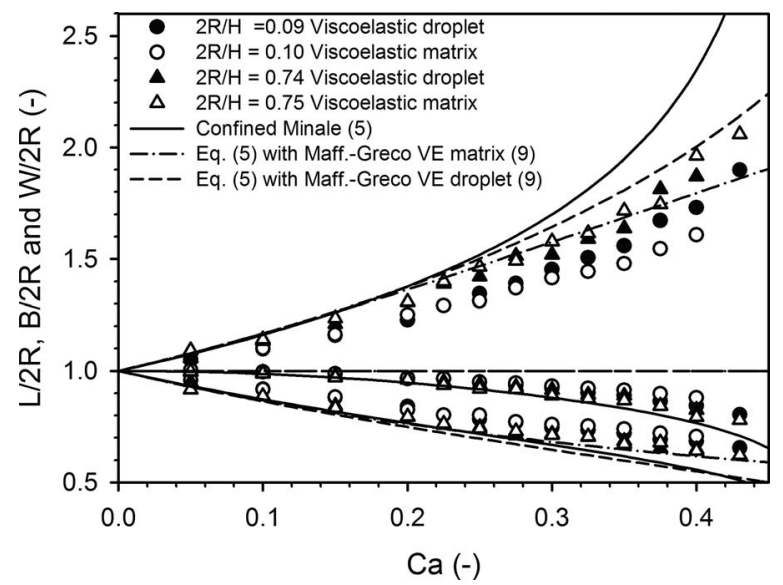

FIG. 14. Droplet axes versus Ca-number at bulk and confined conditions $(\lambda=0.45$ and $\mathrm{De}=1)$.

distinct difference in droplet shape can be noticed between the system with a viscoelastic matrix and that with a viscoelastic droplet. Similar to the results at a viscosity ratio of 1.5 shown in Fig. 13, the viscoelastic droplet shows more tendency toward the formation of sigmoidal droplet shapes. Velocity and pressure fields in and around the droplets are needed to provide insight in the origin of these differences.

\section{CONCLUSIONS}

The effect of confinement on the steady droplet deformation and droplet orientation during shear flow is studied for blends with one viscoelastic component. The experiments are performed with a counter rotating plate-plate device combined with a microscopy setup. The confinement ratio is varied between 0.1 and 0.75 . The studied blends have a viscosity ratio of either 1.5 or 0.45 and the De-number of the viscoelastic phase is kept constant at 1 . As viscoelastic component a model Boger fluid, which is fully rheologically

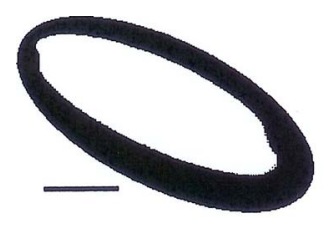

(a) $2 \mathrm{R} / \mathrm{H}=0.11$

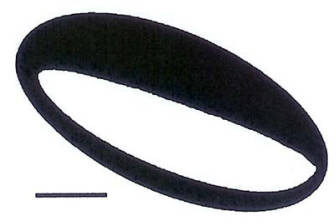

(c) $2 \mathrm{R} / \mathrm{H}=0.09$

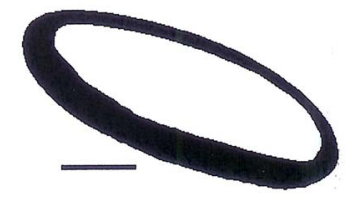

(b) $2 \mathrm{R} / \mathrm{H}=0.74$

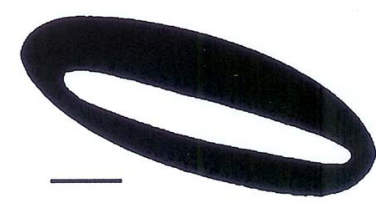

(d) $2 \mathrm{R} / \mathrm{H}=0.75$

FIG. 15. Microscopy images in the velocity-velocity gradient plane for a viscoelastic droplet $(R=137 \mu \mathrm{m}$, top) and a viscoelastic matrix $(R=148 \mu \mathrm{m}$, bottom $)$ at $\mathrm{Ca}=0.35, \lambda=0.45$, and $\mathrm{De}=1$, indicated scale is $100 \mu \mathrm{m}$. 
characterized in both shear and extensional flow [Verhulst et al. (2007a, 2009a)], is used. This makes the experimental data set suitable as a guide for future modeling.

Under bulk conditions, viscoelasticity of the matrix causes a reduction in the droplet deformation compared to the Newtonian reference system. The deformation of a viscoelastic droplet, on the other hand, is similar to that of a Newtonian droplet at the same viscosity ratio. At the applied De-number of 1 , the experimental results indicate that the droplet orientation is hardly affected by the viscoelasticity of the components. The phenomenological Maffettone-Greco [Maffettone and Greco (2004)], Minale [Minale (2004)], and modified Minale models [Minale (2004); Verhulst et al. (2007a)] are used to describe the bulk data. It can be concluded that the different models each provide optimal results for a certain range of the dimensionless parameters. It was however noted by Verhulst et al. (2007a) that the underlying rheological equation, used in the models, is too simplistic to allow a complete quantitative description of the effect of component viscoelasticity on the steady and transient droplet behavior. In addition, at the highest Canumbers, phenomenological models will probably not suffice anymore, causing a need for accurate numerical simulations.

Above a confinement ratio of approximately 0.3 , the presence of the walls starts to influence the droplet behavior. Similar to Newtonian systems, confinement increases the droplet deformation and its orientation toward the flow direction. Both a higher viscosity ratio and droplet viscoelasticity stimulate the formation of sigmoidal droplet shapes at high confinement ratios. Whereas confinement and viscoelasticity effects are independent at a viscosity ratio of 1.5 , matrix viscoelasticity clearly enhances the influence of the walls on the droplet deformation at a lower viscosity ratio. For the present conditions, the deformation of the droplets is not affected by their viscoelasticity, at least up to a confinement ratio of 0.75 . This observation is expected to remain valid as long as the droplet shape is ellipsoidal. The increased orientation, resulting from the confinement of the droplets between the two plates, is similar for all systems studied. From these results and the leveling off of the viscoelasticity effects at higher De-numbers, as reported in Verhulst et al. (2009a), it can be concluded that for the steady droplet shape in shear flow, confinement effects can easily become more pronounced than viscoelasticity effects.

The Shapira-Haber theory [Shapira and Haber (1990)], originally derived for Newtonian droplets in a Newtonian matrix in confined shear flow, is combined here with phenomenological bulk models, in order to incorporate the effects of component viscoelasticity. To that end, the Taylor [Taylor (1934)] bulk deformation parameter is replaced by the deformation parameter obtained from either the Maffettone-Minale [Maffettone and Minale (1998, 1999)], modified Minale [Minale (2004); Verhulst et al. (2007a)], or Maffettone-Greco [Maffettone and Greco (2004)] bulk model. Interestingly, the effect of confinement on the steady state droplet deformation is rather well described under the conditions of the present study, although the effect of viscoelasticity is not explicitly taken into account in the correction factor for wall effects. Nevertheless, although good results are obtained for the deformation parameter, the complete droplet shape, nor the orientation of the droplets, can be predicted with this empirical model. Therefore, more sophisticated models or simulations are needed to describe the droplet dynamics in detail.

\section{ACKNOWLEDGMENTS}

The author (R.C.) is indebted to the Research Foundation-Flanders (FWO) for a Ph.D. Fellowship. This work was partially funded by onderzoeksfonds K.U.Leuven (Grant Nos. GOA03/06 and GOA09/002). 


\section{References}

Aggarwal, N., and K. Sarkar, "Deformation and breakup of a viscoelastic drop in a Newtonian matrix under steady shear," J. Fluid Mech. 584, 1-21 (2007).

Aggarwal, N., and K. Sarkar, "Effects of matrix viscoelasticity on viscous and viscoelastic drop deformation in shear flow," J. Fluid Mech. 601, 63-84 (2008).

Cardinaels, R., A. Vananroye, K. Verhulst, and P. Moldenaers, "Separate and combined influence of confinement and component viscoelasticity on single droplet behavior during shear flow," in Proceedings of the Polymer Processing 24th Annual Meeting (Polymer Processing Society, Salerno, 2008a).

Cardinaels, R., K. Verhulst, and P. Moldenaers, "Drop shape dynamics during shear flow in blends with a viscoelastic component: Bulk and confined conditions," in Proceedings of the 9th European Symposium on Polymer Blends (University of Palermo, Palermo, 2007).

Cardinaels, R., K. Verhulst, Y. Renardy, and P. Moldenaers, "Transient droplet behavior and droplet breakup during bulk and confined shear flow in blends with one viscoelastic component: experiments, modelling and simulations," in Proceedings of the 15th International Congress on Rheology edited by A. Co, L. G. Leal, R. H. Colby, and A. J. Giacomin (American Institute of Physics, Monterey, 2008b), pp. 1405-1407.

Chinyoka, T., Y. Renardy, M. Renardy, and D. B. Khismatullin, "Two-dimensional study of drop deformation under simple shear for Oldroyd-B liquids," J. Non-Newtonian Fluid Mech. 130, 45-56 (2005).

Chung, C., M. A. Hulsen, J. M. Kim, K. H. Ahn, and S. J. Lee, "Numerical study on the effect of viscoelasticity on drop deformation in simple shear flow and 5:1:5 planar contradiction /expansion microchannel," J. Non-Newtonian Fluid Mech. 155, 80-93 (2008).

Dressler, M., and B. J. Edwards, "The influence of matrix viscoelasticity on the rheology of polymer blends," Rheol. Acta 43, 257-282 (2004).

Elmendorp, J. J., and R. J. Maalcke, “A study on polymer blending microrheology,” Polym. Eng. Sci. 25, 1041-1047 (1985).

Greco, F., "Drop deformation for non-Newtonian fluids in slow flows," J. Non-Newtonian Fluid Mech. 107, 111-131 (2002).

Guido, S., and F. Greco, "Drop shape under slow steady shear flow and during relaxation. Experimental results and comparison with theory," Rheol. Acta 40, 176-184 (2001).

Guido, S., and F. Greco, "Dynamics of a liquid drop in a flowing immiscible liquid," in Rheology Reviews 2004, edited by D. M. Binding and K. Walters (The British Society of Rheology, Aberystwyth, 2004), pp. 99-142.

Guido, S., M. Simeone, and F. Greco, "Effects of matrix viscoelasticity on drop deformation in dilute polymer blends under slow shear flow," Polymer 44, 467-471 (2003a).

Guido, S., M. Simeone, and F. Greco, "Deformation of a Newtonian drop in a viscoelastic matrix under steady shear flow-Experimental validation of slow flow theory," J. Non-Newtonian Fluid Mech. 114, 65-82 (2003b).

Guido, S., M. Simeone, and M. Villone, "Diffusion effects on the interfacial tension of immiscible polymer blends," Rheol. Acta 38, 287-296 (1999).

Janssen, P. J. A., and P. D. Anderson, "Boundary-integral method for drop deformation between parallel plates," Phys. Fluids 19, 043602 (2007).

Khismatullin, D., Y. Renardy, and M. Renardy, "Development and implementation of VOF-PROST for 3D viscoelastic liquid-liquid simulations," J. Non-Newtonian Fluid Mech. 140, 120-131 (2006).

Lerdwijitjarud, W., R. G. Larson, A. Sirivat, and M. J. Solomon, "Influence of weak elasticity of dispersed phase on droplet behaviour in sheared polybutadiene/poly(dimethylsiloxane)blends," J. Rheol. 47, 37-58 (2003).

Lerdwijitjarud, W., A. Sirivat, and R. G. Larson, "Influence of dispersed-phase elasticity on steady-state deformation and breakup of droplets in simple shearing flow of immiscible polymer blends," J. Rheol. 48, 843-862 (2004).

Maffettone, P. L., and F. Greco, "Ellipsoidal drop model for single droplet dynamics with non-Newtonian fluids," J. Rheol. 48, 83-100 (2004).

Maffettone, P. L., F. Greco, M. Simeone, and S. Guido, "Analysis of start-up dynamics of a single drop though an ellipsoidal drop model for non-Newtonian fluids," J. Non-Newtonian Fluid Mech. 126, 145-151 (2005). 
Maffettone, P. L., and M. Minale, "Equation of change for ellipsoidal drops in viscous flow," J. Non-Newtonian Fluid Mech. 78, 227-241 (1998)

Maffettone, P. L., and M. Minale, "Erratum,” J. Non-Newtonian Fluid Mech. 84(1), 105-106 (1999).

Mighri, F., and P. J. Carreau, "Influence of elastic properties on drop deformation and breakup in shear flow," J. Rheol. 42, 1477-1490 (1998).

Minale, M., "Deformation of a non-Newtonian ellipsoidal drop in a non-Newtonian matrix: Extension of Maffettone-Minale model," J. Non-Newtonian Fluid Mech. 123, 151-160 (2004).

Minale, M., "A phenomenological model for wall effects on the deformation of an ellipsoidal drop in viscous flow," Rheol. Acta 47, 667-675 (2008).

Renardy, Y., "The effects of confinement and inertia on the production of droplets," Rheol. Acta 46, 521-529 (2007).

Shapira, M., and S. Haber, "Low Reynolds number motion of a droplet in shear flow including wall effects," Int. J. Multiphase Flow 16, 305-321 (1990).

Sibillo, V., S. Guido, F. Greco, and P. L. Maffettone, "Single drop dynamics under shearing flow in systems with a viscoelastic phase," Macromol. Symp. 228, 31-39 (2005).

Sibillo, V., M. Simeone, S. Guido, F. Greco, and P. L. Maffettone, "Start-up and retraction dynamics of a Newtonian drop in a viscoelastic matrix under simple shear flow," J. Non-Newtonian Fluid Mech. 134, 27-32 (2006a).

Sibillo, V., G. Pasquariello, V. Simeone, V. Cristini, and S. Guido, "Drop deformation in microconfined shear flow," Phys. Rev. Lett. 97, 054502 (2006b).

Taylor, G. I., "The formation of emulsions in definable fields of flow," Proc. R. Soc. London, Ser. A 146, 501-523 (1934).

Tucker, C. L., and P. Moldenaers, "Microstructural evolution in polymer blends," Annu. Rev. Fluid Mech. 34, 177-210 (2002).

Van Puyvelde, P., A. Vananroye, R. Cardinaels, and P. Moldenaers, "Review on morphology development of immiscible blends in confined shear flow," Polymer 51, 5363-5372 (2008).

Vananroye, A., R. Cardinaels, P. Van Puyvelde, and P. Moldenaers, "Effect of confinement and viscosity ratio on the dynamics of single droplets during transient shear flow," J. Rheol. 52, 1459-1475 (2008a).

Vananroye, A., P. J. A. Janssen, P. D. Anderson, P. Van Puyvelde, and P. Moldenaers, "Microconfined equiviscous droplet deformation: Comparison of experimental and numerical results," Phys. Fluids 20, 013101 (2008b).

Vananroye, A., P. Van Puyvelde, and P. Moldenaers, "Effect of confinement on the steady-state behaviour of single droplets during shear flow," J. Rheol. 51, 139-153 (2007).

Verhulst, K., P. Moldenaers, and M. Minale, "Drope shape dynamics of a Newtonian drop in a non-Newtonian matrix during transient and steady shear flow," J. Rheol. 51, 261-273 (2007a).

Verhulst, K., R. Cardinaels, and P. Moldenaers, "Steady state droplet deformation and orientation during shear flow in blends with a viscoelastic component: Bulk and confined conditions," in Proceedings of the Polymer Processing Society Europe/Africa Regional Meeting, Göteborg (Polymer Processing Society, Göteborg, 2007b).

Verhulst, K., R. Cardinaels, Y. Renardy, and P. Moldenaers, "Steady state droplet deformation and orientation during bulk and confined shear flow in blends with one viscoelastic component: Experiments, modeling and simulations," in Proceedings of the 15th International Congress on Rheology, Monterey, edited by A. Co, L. G. Leal, R. H. Colby, and A. J. Giacomin (American Institute of Physics, Monterey, 2008), pp. 1000-1002.

Verhulst, K., R. Cardinaels, P. Moldenaers, Y. Renardy, and S. Afkhami, "Influence of viscoelasticity on drop deformation and orientation in shear flow: Part 1. Stationary states," J. Non-Newtonian Fluid Mech. 156, 29-43 (2009a).

Verhulst, K., R. Cardinaels, P. Moldenaers, S. Afkhami, and Y. Renardy, "Influence of viscoelasticity on drop deformation and orientation in shear flow. Part 2: Dynamics," J. Non-Newtonian Fluid Mech. 156, 44-57 (2009b).

Vrentas, J. S., D. C. Venerus, and C. M. Vrentas, "An exact analysis of reservoir effects for rotational viscosimeters," Chem. Eng. Sci. 46, 33-37 (1991).

Yu, W., M. Bousmina, C. Zhou, and C. L. Tucker, "Theory for drop deformation in viscoelastic systems," J. 
Rheol. 48, 417-438 (2004).

Yu, W., C. Zhou, and M. Bousmina, "Theory of morphology evolution in mixtures of viscoelastic immiscible components,” J. Rheol. 49, 215-236 (2005).

Yue, P., J. J. Feng, C. Liu, and J. Shen, "Viscoelastic effects on drop deformation in steady shear," J. Fluid Mech. 540, 427-437 (2005). 